(Supporting Information)

\title{
Fluorescence Lifetime Imaging of Nanoflares for mRNA Detection in Living Cells
}

\author{
Jing Shi ${ }^{a}$, Ming Zhou ${ }^{* a, b}$, Aihua Gong ${ }^{c}$, Qijun $i^{a}{ }^{a}$, Qian Wu ${ }^{a}$, Gary J. Cheng ${ }^{b}$, \\ Mingyang Yang ${ }^{a}$, Yaocheng Sun ${ }^{c}$
}

[*] Ming Zhou*

State Key Laboratory of Tribology, Tsinghua University, Beijing 100084, People’s

Republic of China

Email: Ming Zhou (zhouming@mail.tsinghua.edu.cn )

\section{Table of Contents:}

Experimental Section

Figure S1. Absorption spectra of AuNPs before and after DNA functionalization.

Figure S2. Evaluation of ex8-flares on nanoflares.

Figure S3. Characterization of the stability of nanoflares against Dnase I

Figure S4. Characterization of the stability of nanoflares against GSH

Figure S5. Kinetic study of nanoflares

Table S1. Representative data of fluorescence lifetimes analysis of cy5 in ex8-flares, nanoflares and non-complementary nanoflares treated cells and nanoflares treated cells of BRCA1 gene knockdown. 


\section{Preparation of Gold nanoparticle}

Gold nanoparticles were prepared by sodium citrate acid reduction method according to published procedures with modification ${ }^{(1)} .1 \mathrm{~mL} \mathrm{HAuCl}_{4}$ solution $(1 \%$, w/v) was diluted in $99 \mathrm{~mL}$ double-stilled water in a conical flask and heated to boiling. $2 \mathrm{~mL}$ trisodium citrate $(1 \%, \mathrm{w} / \mathrm{v})$ was added to the boiling $\mathrm{HAuCl}_{4}$ under vigorous stirring. The mixed solution was kept boiling for 10 minutes under stirring and then cooled to room temperature. BSPP (30mg) was added to the Au colloidal solution (100ml) and the mixture was shaken at room temperature overnight to modify gold nanoparticles. The resulting mixture was centrifuged with a $30 \mathrm{kDa}$ MWCO Millipore Centrifugation filter at the speed of $8000 \mathrm{rpm}$ for $10 \mathrm{~min}$ and the concentrated Au colloid solution in the column was carefully pipetted out for experiment. The concentration of Au colloid was determined by the UV-Vis spectroscopy according to the optical absorbance at $\sim 523 \mathrm{~nm}\left(\varepsilon=2.7 \times 10^{8} \mathrm{~L} \mathrm{~mol}^{-1} \mathrm{~cm}^{-1}\right)$.

\section{Synthesis and Characterization of Oligonucleotides}

The oligonucleotides were synthesized by Sangon Biotech and were used without further purification. The oligonucleotide sequences used in the study were as follows: ex8-recognition, 5'-TCAAT TCAAT GTAGA CAGAC GTCTT TTGAG GTTTT

TT-thiol-3' (Bolded region indicates sequences that is complementary to the sequences of target mRNA); ex8-flare, 5'-cy5-CCTCA AAAGA CGTCT G-3'; ex8-target; 5'-CCTCA AAAGA CGTCT GTCTA CATTG AATTG A-3'; 
non-complementary recognition: 5'-CCCTG TTAGT CGTAG CAGAC GTCTT TTGAG GTTTT TT-thiol-3’.

\section{Preparation of nanoflares}

The conjugates of AuNP-DNA were prepared following a regular protocol. The thiol modified oligonucleotides $(100 \mu \mathrm{M})$ were incubated with TCEP $(100 \mathrm{mM})$ in a ratio of 1:200 for one hour to reduce disulfide bond to monothiol, then 1nM AuNP solutions were added to the treated oligonucleotide solutions with ex8-recognition in a molar ration of 1:100 for AuNP and was shaken for two hours. Then 10×PBS buffer and 1\% SDS were added to achieve a $1 \times \mathrm{PBS}$ and $0.1 \%$ SDS concentration. $\mathrm{NaCl}$ solution (1M) was subsequently added to the solution every four hours with an increment of $50 \mathrm{mM}$ to the final concentration of $300 \mathrm{mM}$ and the mixture was then shaken overnight. The AuNP-ex8-recognition conjugate solution was washed three times with $1 \times$ PBS buffer in $30 \mathrm{kDa}$ MWCO Millipore Centrifugation filter to remove unattached oligonucleotides and redispersed in $1 \times$ PBS buffer. The red shift of the absorption spectra of the AuNPs before and after the incubation with ex8-recogntion sequences indicated the successful attachment of recognition oligonucleotides (Fig. S1). Concentration of Au colloid was determined by the UV-Vis spectroscopy according to the optical absorbance at $\sim 523 \mathrm{~nm}$. 20nM AuNP-ex8-recognition conjugate was mixed with ex8-flare in 1:100 molar ratio and incubated at $70^{\circ} \mathrm{C}$ for 1 hour and then cooled to room temperature to allow hybridization. After the annealing procedure, the product was washed with $1 \times$ PBS buffer in $100 \mathrm{kDa}$ MWCO Millipore 
Centrifugation filter at the speed of $8000 \mathrm{rpm}$ for $5 \mathrm{~min}$ to remove unattached ex 8 -flare and resuspended in $1 \times$ PBS buffer. The concentration of Au colloid was determined by the UV-Vis spectroscopy and the solution was stored at $4^{\circ} \mathrm{C}$.

\section{Characterization of Nanoflares}

A fluorescence based method was used for quantitation of the surface coverage of ex8-flare loaded on AuNPs ${ }^{(2)}$. The nanoflares were incubated with $2 \mathrm{mM}$ mercaptoethanol overnight at room temperature. Then the solution was centrifuged and the supernant were collected for fluorescence measurement. The fluorescence was measured with F-7000 (HITACHI) fluorescence spectrometer. The solution was excited at $635 \mathrm{~nm}$, and the emission intensity at $670 \mathrm{~nm}$ was interpolated into a standard linear curve calibrated with known concentration of cy5-modifed DNA（Fig S2).The average value and standard deviation were obtained by three parallel experiments.

\section{Fluorescence Lifetime measurements of nanoflares in solution}

Nanoflares were diluted to $1 \mathrm{nM}$ in $1 \times \mathrm{PBS}$ with a concentration of $0,5,10,20,40$, 80, 100, 150, 200nM ex8-target DNA and non-target DNA. Flares were diluted to $1 \mathrm{nM}$ in $1 \times \mathrm{PBS}$. All of the fluorescence lifetimes were recorded on a Picoquant PicoHarp 300 TCSPC module exciting at $640 \mathrm{~nm}$ and measuring emission at $690 \pm$ $35 \mathrm{~nm}$. Samples were dropped on to coverslip processed by Piranha solution and illuminated by a multichannel picosecond diode laser (PDL 828 "Sepia II") through a $60 \times$ silicone oil microscope objective. The lifetime values were analyzed with 
measured instrument response function (IRF) deconvolved from the signal using Fluofit software. At least three parallel experiments were performed.

\section{Fluorescence Intensity measurements of nanoflares in solution}

Fluorescence spectroscopy (HITACHI, F-7000) was used to quantify the fluorescence signal of a fixed concentration of nanoflares with different nanomoles of ex8-target and non-target sequences. Nanoflares were diluted to a concentration of $1 \mathrm{nM}$ in $1 \times$ PBS buffer treated with $0,5,10,20,40,80,100,150,200 \mathrm{nM}$ ex8-target and non-target sequences. After equilibrated for $30 \mathrm{~min}$ at room temperature, samples were excited at $635 \mathrm{~nm}$, emission fluorescence signals at $670 \mathrm{~nm}$ were recorded respectively.

\section{The study of the stability against DNase and GSH of nanoflares}

For the study of nuclease degradation effects, nanoflares were diluted in $1 \times \mathrm{PBS}$ to a concentration of $1 \mathrm{nM}$ at room temperature and equilibrated for $10 \mathrm{~min}$ before $1 \mathrm{ul}$ of 50 unit/ml DNase I was added. The lifetime decay curve was measured every $5 \mathrm{~min}$ for 2 hours. For the GSH effects, nanoflares were diluted in $1 \times$ PBS to a concentration of $1 \mathrm{nM}$ at room temperature. The lifetime of the sample was monitored every $5 \mathrm{~min}$ for 2 hours immediately after $2 \mathrm{mM}$ GSH was added. Both samples were put into a Chambered cover glass and illuminated by a multichannel picosecond diode laser (PDL 828 "Sepia II") through a $60 \times$ silicone oil microscope objective. More than 10,000 photons were collected for analysis each sample. The instrument response function was measured and used for fluorescence lifetime deconvolution approximation using Fluofit software. 


\section{The study of the kinetics of the nanoflares}

Nanoflares were diluted in $1 \times$ PBS to a concentration of $1 \mathrm{nM}$ at room temperature. As $200 \mathrm{nM}$ target sequences were added to nanoflares, fluorescence lifetime of the solution was monitored every 5 minutes for 2 hours. The fluorescence intensity was also monitored with the same procedure with fluorescence spectrometer.

\section{Cell Culture}

Human breast cancer cell lines MDA-MB-231 purchased from The Culture Collection of the Chinese Academy of Sciences (Shanghai) were grown in L-15 medium (GIBCO) with $10 \%$ fetal bovine serum and incubated in $100 \%$ air at $37^{\circ} \mathrm{C}$. The prepared nanostructures were diluted in fresh medium in $1 \mathrm{nM}$ concentration and then sterilized using $0.2 \mathrm{um}$ acetate syringe filter (Acrodisc) to prevent contamination.

\section{mRNA knockdown and RT-PCR}

The siRNA and the primer were designed and synthesized by Genepharma and the sequences used in the study were as follows. SiRNA sense (5'-3'), CCUUC UAACA GCUAC CCUUT T. SiRNA antisense (5'-3'), AAGGG UAGCU GUUAG AAGGT T. Primer, BRCA1 forward (5'-3'), GTATT CTGAG AGGCT GCTGC T. BRCA1 reverse (5'-3'), TACCC AGAGC AGAGG GTGAA. GADPH forward, GGTGA AGGTC GGTGT GAACG. GADPH reverse, CTCGC TCCTG GAAGA TGGTG. SiRNA were transfected to MDA-MB-231 cells by Lipofectmine3000 (Life Technologies) and incubated for $48 \mathrm{~h}$ following the protocol. The cells were then 
harvested, and RNA in cells were extracted following a regular Trizol method. cDNA synthesis was conducted using Reverted First Strand cDNA Synthesis Kit (Thermo Scientific). Relative level of BRCA1 mRNA was estimated from the quantity of mRNA PCR products using a semi-quantitative method.

\section{Cell Imaging and FLIM Experiments}

Cells were cultured for $24 \sim 48$ hours before treatment and then incubated with nanoflares and non-complementary nanoflares for 2.5 hours respectively. Ex8-flares were transfected with Lipofectamine3000 (Life Technologies) following its protocol and incubated for 2.5 hours. All of the laser confocal images were recorded with an Olympus FluoView1200 laser confocal microscopy at $40 \times$ magnification using a HeNe laser excitation source at $633 \mathrm{~nm}$ and $405 \mathrm{~nm}$. Lifetime images were obtained with a Picoquant PicoHarp 300 TCSPC module connected to it at $40 \times$ magnification as well. All the fluorescence-intensity images obtaining settings were held constant throughout the experiment. More than 50,000 photons were collected for each lifetime images and calculation.

\section{Flow Cytometry}

Cells were incubated in 6 well plates for 24 hours before treatment. Cells were treated with the same protocol as that of samples for imaging. After 2 hours' nanoflare(1nM) treatment, Cells were trypsinized, and washed twice with $1 \times$ PBS and resuspended in $1 \times$ PBS . ACEA Novocyte Flow Cytometer (ACEA Biosciences, Inc.) was used for cytometric analysis. The fluorescence of each cell was recorded in cy5 channel and all 
settings were the same throughout all experiments. 10,000 cells were analyzed in each well and the experiment was done in three replicates. 


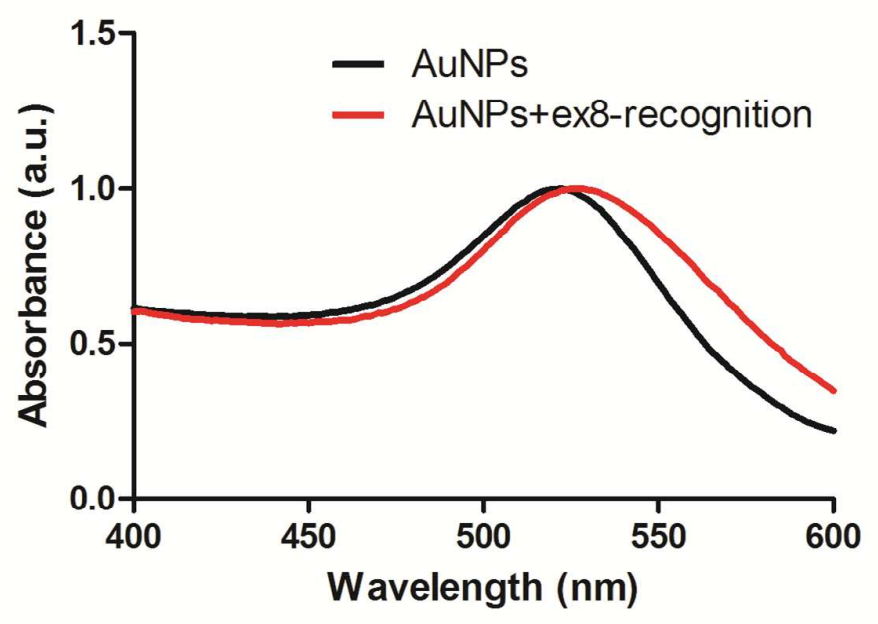

Figure S1. Absorption spectra of AuNPs before and after DNA functionalization. The red shift of the absorbance peak indicated the successful coating of ex8-recognition sequences.
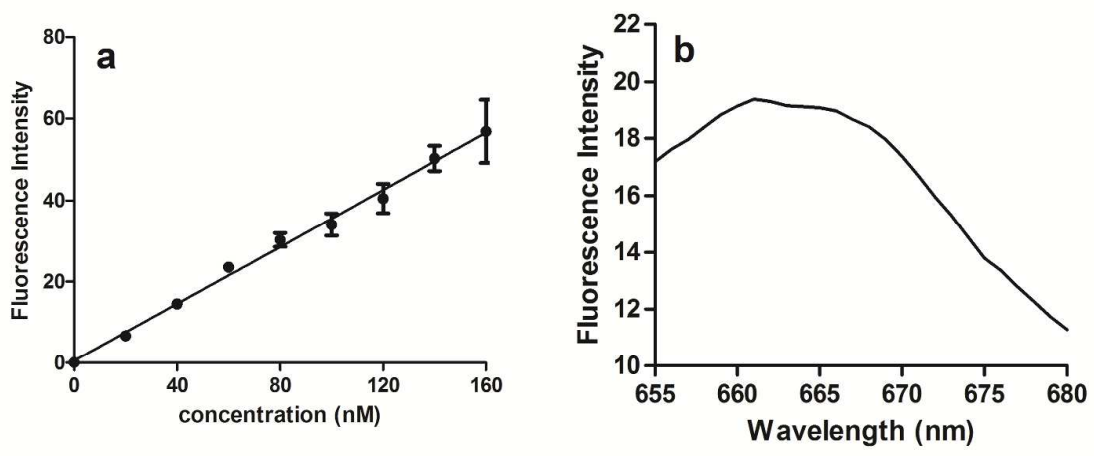

Figure S2. Evaluation of ex8-flares on nanoflares. (a) Standard linear calibration curve of fluorescence signal of nanoflares was established by measuring a series of ex8-flares of known concentration. (b) Fluorescence signal of mercaptoethanol treated nanoflares. 

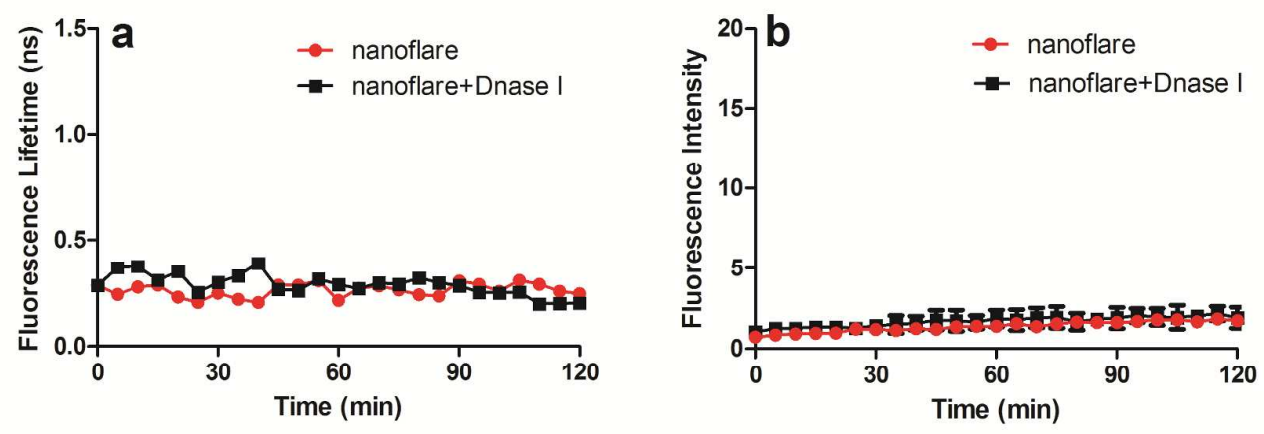

Figure S3. Characterization of the stability of nanoflares against Dnase I.

(a) Fluorescence lifetime measurement. (b) Fluorescence intensity measurement.
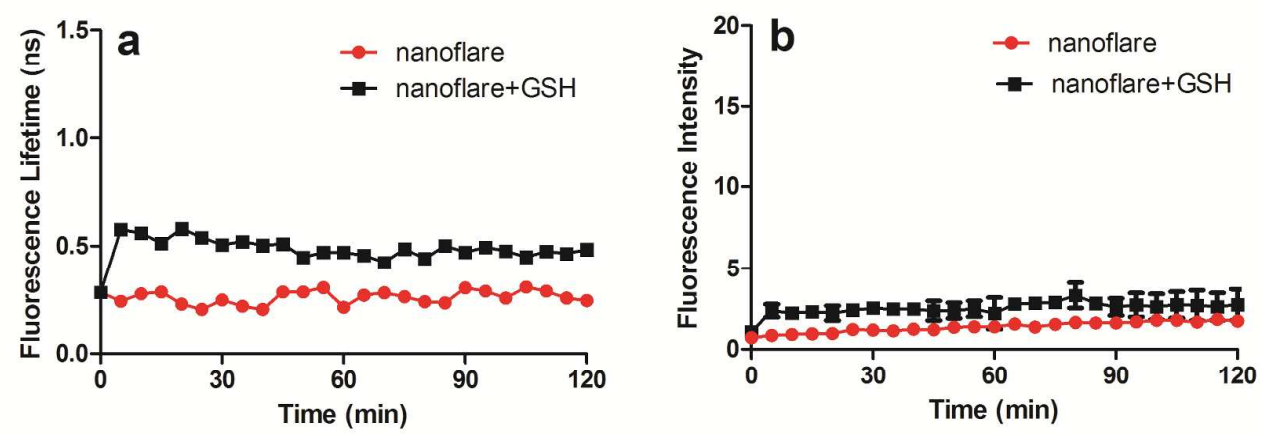

Figure S4. Characterization of the stability of nanoflares against glutathione(GSH).

(a) Fluorescence lifetime measurement. (b) Fluorescence intensity measurement.
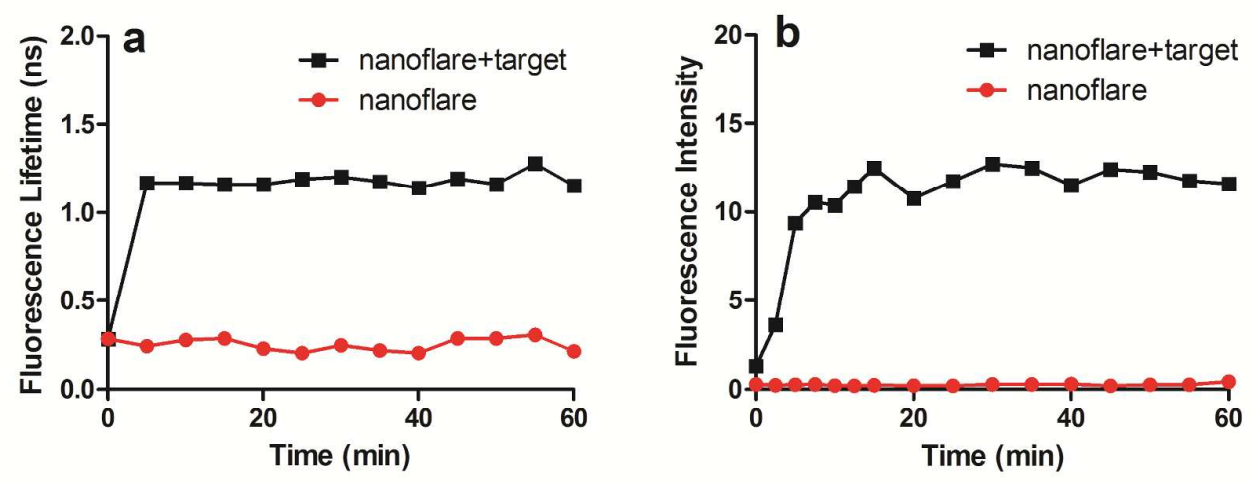

Figure S5. Kinetic study of nanoflares.

(a) Fluorescence lifetime measurement. (b) Fluorescence intensity measurement. 
Table S1 Representative data of fluorescence lifetimes analysis of cy5 in ex8-flares, nanoflares and non-complementary nanoflares treated cells and nanoflares treated cells with BRCA1 gene knockdown.

\begin{tabular}{|c|c|c|c|c|c|c|c|c|}
\hline Treatment & $\mathrm{A}_{1}$ & $\mathrm{~A}_{2}$ & $A_{3}$ & $\tau_{1}$ & $\tau_{2}$ & $\tau_{3}$ & $x^{2}$ & $\tau$ av \\
\hline Ex8-flare & $37.84(0.69)$ & $10.5(0.7)$ & -- & $2.3734(0.008)$ & $1.421(0.038)$ & -- & 1.050 & 2.166 \\
\hline NF & $32.26(0.23)$ & $13.88(0.19)$ & -- & $2.441(0.0045)$ & $1.299(0.013)$ & -- & 1.049 & 2.098 \\
\hline Noncom-NF & $20.02(0.31)$ & $17.17(0.23)$ & $6.49(0.17)$ & $2.422(0.006)$ & $1.44(0.02)$ & $0.353(0.0097)$ & 0.971 & 1.73 \\
\hline $\begin{array}{l}\text { NF ( siRNA } \\
\text { treated cells) }\end{array}$ & $16.93(0.19)$ & $20.03(0.56)$ & $6.37(0.54)$ & $2.602(0.007)$ & $1.67(0.04)$ & $0.613(0.013)$ & 0.997 & 1.8767 \\
\hline
\end{tabular}

NF is short for nanoflares. Noncom-NF is short for non-complementary nanoflares.

\section{References:}

(1). Taton, T. A. Current Protocols in Nucleic Acid Chemistry 2002, 12-2.

(2). Demers, L. M.; Mirkin, C. A.; Mucic, R. C.; Reynolds, R. A.; Letsinger, R. L.; Elghanian, R.; Viswanadham, G. Anal Chem 2000, 72, (22), 5535-5541. 